OPEN ACCESS

Edited by:

Miguel Alejandro Mostafa,

Pennsylvania State University,

United States

Reviewed by:

Dmitri Semikoz,

UMR7164 Astroparticule et Cosmologie (APC), France

Kumiko Kotera,

UMR7095 Institut d'Astrophysique de

Paris (IAP), France

Karl-Heinz Kampert,

University of Wuppertal, Germany

*Correspondence:

Francis Halzen

halzen@icecube.wisc.edu Ali Kheirandish

akheirandish@icecube.wisc.edu

Specialty section:

This article was submitted to

High-Energy and Astroparticle

Physics,

a section of the journal

Frontiers in Astronomy and Space

Sciences

Received: 12 December 2018

Accepted: 15 April 2019

Published: 03 May 2019

Citation:

Halzen F and Kheirandish A (2019)

Multimessenger Search for the

Sources of Cosmic Rays Using

Cosmic Neutrinos.

Front. Astron. Space Sci. 6:32.

doi: 10.3389/fspas.2019.00032

\section{Multimessenger Search for the Sources of Cosmic Rays Using Cosmic Neutrinos}

\author{
Francis Halzen * and Ali Kheirandish* \\ Department of Physics \& Wisconsin IceCube Particle Astrophysics Center, UW-Madison, Madison, WI, United States
}

After updating the status of the measurements of the cosmic neutrino flux by the lceCube experiment, we summarize the evidence for the first identified source of cosmic rays and speculate on the connection between the two observations.

Keywords: multimessenger, neutrinos, cosmic rays, blazars, cosmic accelerators

\section{DETECTING COSMIC NEUTRINOS}

The highest energy radiation reaching us from the universe is not radiation at all; it is cosmic rays, high-energy nuclei that are mostly protons, except possibly at the end of the spectrum. We do not know where they come from or how they are accelerated to such extreme energies. However, with the compelling evidence for a distant blazar as the source of a very high energy muon neutrino, neutrinos have provided a breakthrough in solving this puzzle (Aartsen et al., 2018a,b).

The rationale for searching for cosmic ray sources by observing neutrinos is straightforward: in the vicinity of neutron stars and black holes, the gravitational energy released in the accretion of matter accelerates protons or heavier nuclei, which subsequently interact with gas or ambient radiation to produce neutrinos originating from the decay of pions and other secondary particles ${ }^{1}$. When accelerated, protons interact with photons; for instance, both neutral and charged pion secondaries are produced. While charged pions decay into three high-energy neutrinos and antineutrinos, neutral pions decay and create a flux of high-energy gamma rays. These photons, referred to as pionic photons, are distinguished from photons radiated by electrons that may be accelerated along with the cosmic rays. Pionic photons are what provide the motivation for multimessenger observations.

The search for high-energy astrophysical neutrinos led to the development of large scale detectors: DUMAND in the Pacific Ocean, NT200 in Lake Baikal, AMANDA at the South Pole, and ANTARES in the Mediterranean Sea. These detectors use highly transparent natural water or ice as a Cherenkov medium that is then instrumented with photomultipliers; see (Katz and Spiering, 2012). The Cherenkov light patterns radiated by secondary charged particles produced by neutrinos interacting inside or near the instrumented volume encode the flavor, energy, and arrival direction of the neutrino. Based on the expectation that cosmic accelerators may produce similar energies in cosmic rays, gamma rays, and neutrinos, it had been anticipated that the construction of a cubic-kilometer, gigaton-scale detector would be sufficient to make a first detection of high-energy neutrinos of cosmic origin.

The IceCube Neutrino Observatory (Aartsen et al., 2017c) transformed one cubic kilometer of natural ice into a Cherenkov detector, operating as the world's premier neutrino detector above energies of approximately $10 \mathrm{GeV}$. IceCube was constructed between 2004 and 2010 near the

\footnotetext{
${ }^{1}$ It is worth mentioning that other scenarios could lead to particle acceleration, such as Poynting flux in neutron stars.
} 
Amundsen-Scott South Pole Station, Antarctica, at the geographic South Pole. Below a depth of $1,450 \mathrm{~m}$, a cubickilometer of glacial ice is instrumented with 86 cables called "strings," each of which is equipped with 60 digital optical modules (DOMs). The DOM is a glass pressure vessel containing a 10-inch photomultiplier tube (PMT) and digitizing electronics which capture the PMT light signal (Aartsen et al., 2017c). These digitized waveforms are the basis of IceCube analysis: the recorded arrival time and amplitude of the photons are used to reconstruct the direction, energy, and location of particle interactions in the ice. The central strings are more densely spaced, forming the DeepCore component of IceCube, and most DOMs in this region are equipped with high-quantum-efficiency PMTs to detect lower energy particle interactions. IceCube also includes a surface cosmic ray air shower detector, IceTop, that consists of 81 stations, each comprising two light-tight tanks of (frozen) water instrumented with two DOMs each.

The deep ice itself constitutes the detector, forming both support structure and Cherenkov medium. Its optical properties, scattering and absorption, are depth-dependent, predominantly due to layers of dust resulting from past glacial-maximum climate events, with scattering in the glacier being anisotropic (Aartsen et al., 2013b). Additionally, scattering results from bubbles in the re-frozen "hole ice" in which the strings have been deployed. Each DOM is equipped with LED flashers that are used as calibration light sources to determine the optical properties of the natural ice. The high statistics data sets, as well as extensive calibration data, have enabled sophisticated modeling of the optics of the ice.

In principle, the particle trajectory is determined by the arrival times of photons at the optical sensors, while the number of photons observed is used to determine the energy deposited by charged secondary particles in the detector. For illustration, the Cherenkov patterns initiated by an electron (or tau) neutrino of $1 \mathrm{PeV}$ energy and a neutrino induced muon losing 2.6 PeV energy while traversing the detector are contrasted in Figure 1. For the cascade (shower) event shown in the left panel of Figure 1, more than 300 digital optical modules (DOMs) report a total of more than 100,000 photoelectrons.

Long tracks resulting from muon neutrino interactions can be pointed back to their sources with $\mathrm{a} \leq 0.4^{\circ}$ angular resolution. In contrast, the reconstruction of cascade directions, in principle currently possible to a few degrees, is still in the development stage in IceCube, where originally it was limited to within $10^{\circ} \sim 15^{\circ}$ of the direction of the incident neutrino (Aartsen et al., 2014; Yuan, 2017). Determining the deposited energy from the observed light pool is, however, relatively straightforward, and a resolution of better than $15 \%$ is possible; the same value holds for the reconstruction of the energy deposited by a muon track inside the detector.

IceCube operates continuously, and monitors the whole sky simultaneously, in the search for high-energy neutrinos of cosmic origin, collecting very high statistics of cosmic ray muons and atmospheric neutrino data sets. Detected neutrino energies cover more than six orders of magnitude, from $5 \mathrm{GeV}$ in DeepCore to beyond $5 \mathrm{PeV}$. Soon after the completion of the detector, with 2 years of data, IceCube discovered an extragalactic flux of cosmic neutrinos with an energy density in the local Universe that is similar to that in gamma rays (See Ackermann et al., 2015; Fang and Murase, 2018).

Two principal methods are used to identify cosmic neutrinos. The first method has traditionally focused on the observation of muon neutrinos that interact primarily outside the detector. High-energy muon neutrinos interact and produce kilometerlong muon tracks passing through the instrumented volume. Although this allows identifying neutrinos that interact outside the detector, it relies on using the Earth as a filter in order to remove the background of cosmic-ray muons. This method limits the neutrino view to a single flavor and half the sky. IceCube has measured the atmospheric neutrino background flux over more than 5 orders of magnitude in energy with
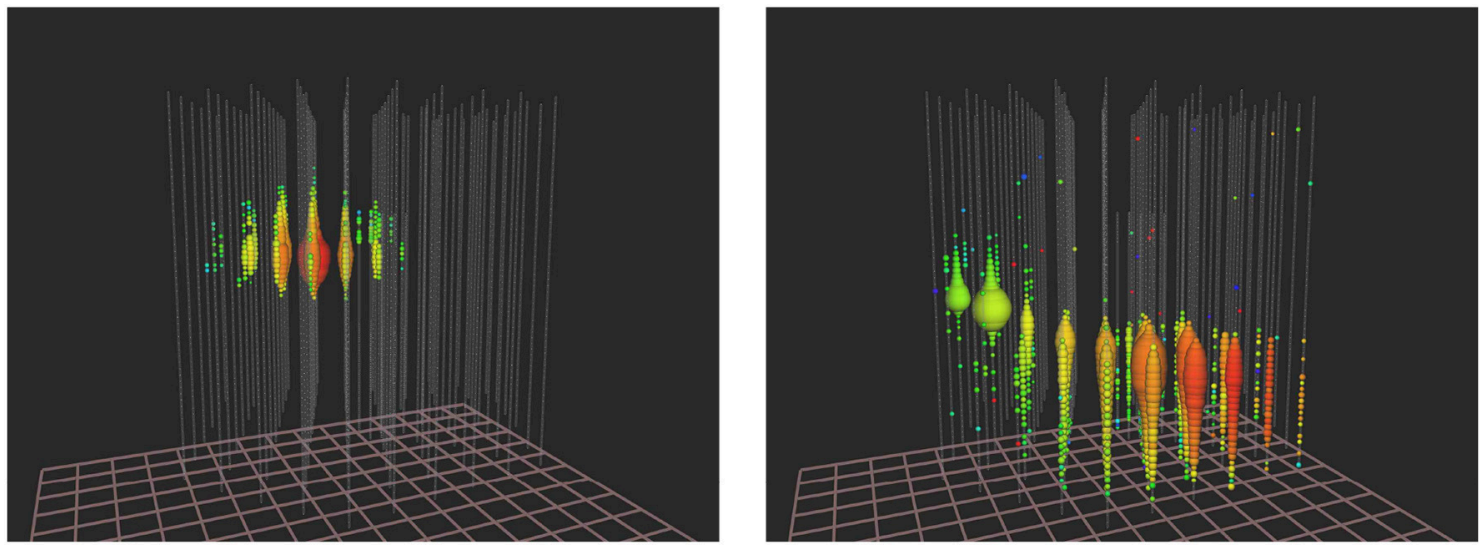

FIGURE 1 | (Left) Light pool produced in IceCube by a shower initiated by an electron or tau neutrino. The measured energy is 1.14 PeV, which represents a lower limit on the energy of the neutrino that initiated the shower. White dots represent sensors with no signal. For the colored dots, the color indicates arrival time, from red (early) to purple (late) following the rainbow, and size reflects the number of photons detected. (Right) An upgoing muon track traverses the detector at an angle of $11^{\circ}$ below the horizon. The deposited energy, i.e., the energy equivalent of the total Cherenkov light of all charged secondary particles inside the detector, is 2.6 PeV. Image: IceCube Collaboration, permission: Elsevier. 
a result that is consistent with theoretical calculations. With 8 years of data, IceCube has observed an excess of events at energies beyond $100 \mathrm{TeV}$ (Aartsen et al., 2015, 2016a, $2017 b)$ that cannot be accounted for by the atmospheric flux. Even acknowledging large uncertainties in the extrapolation of the atmospheric component to higher energy, the statistical significance of the excess astrophysical flux is $6.7 \sigma$. Although the detector only records the energy of the secondary muons inside the detector, from Standard Model physics we can infer the energy spectrum of the parent neutrinos. The resulting high-energy cosmic muon neutrino flux is well-described by a power law with a spectral index of $2.19 \pm 0.10$ and a normalization at $100 \mathrm{TeV}$ neutrino energy of $\left(1.01_{-0.23}^{+0.26}\right) \times$ $10^{-18} \mathrm{GeV}^{-1} \mathrm{~cm}^{-2} \mathrm{sr}^{-1}$ (Aartsen et al., 2017b).

An alternative method exclusively identifies high-energy neutrinos interacting inside the detector, so-called high-energy starting events (HESE). It divides the instrumented volume of ice into an outer veto shield and a $\sim$ 500-megaton inner fiducial volume. The advantage of focusing on neutrinos interacting inside the instrumented volume of ice is that the detector functions as a total absorption calorimeter (Aartsen et al., 2014). With this method, neutrinos from all directions in the sky and all flavors can be identified, which includes both muon tracks as well as secondary showers, produced by charged-current interactions of electron and tau neutrinos, and neutral current interactions of neutrinos of all flavors. Furthermore, this method can separate neutrinos of atmospheric and cosmic origin. Atmospheric neutrinos reaching us from the Southern Hemisphere are readily distinguishable because they are accompanied by particles produced in the same air shower where the neutrinos originate. They are further separable from cosmic neutrinos by their well-measured energy. A sample event with a light pool of roughly one hundred thousand photoelectrons extending over more than $500 \mathrm{~m}$ is shown in the left panel of Figure 1.

The advantages of these two methods of separating cosmic neutrinos from the atmospheric backgrounds are complementary. It was the HESE method that succeeded in revealing the first evidence for cosmic neutrinos (Aartsen et al., 2013a,c). Observed events with $\mathrm{PeV}$ energies, and no trace of accompanying muons from an atmospheric shower, are highly unlikely to be of atmospheric origin. The 7-year data set contains a total of 60 neutrino events with deposited energies ranging from $60 \mathrm{TeV}$ to $10 \mathrm{PeV}$. The data are consistent with an astrophysical component with a spectrum close to $E^{-2}$ above an energy of $\sim 200 \mathrm{TeV}$.

We should mention here that there is yet another method to conclusively identify cosmic neutrinos: the observation of very high energy tau neutrinos. Tau neutrinos above about $100 \mathrm{TeV}$ produce two spatially separated showers in the detector, one from the interaction of the tau neutrino and the second one from the tau decay; the mean tau lepton decay length is about $50 \mathrm{~m} / \mathrm{PeV}$. Two such candidate events have been recently identified (Taboada, 2018). In addition to the double cascade candidates, we should also mention that a first candidate event has been attributed to the Glashow resonance. This event was identified in a search for partially contained events, and represents $\sim 6 \mathrm{PeV}$ in energy deposited in the detector; See Figure 2 (Aartsen et al., 2017b; Lu, 2018).

\section{ICECUBE NEUTRINOS AND FERMI PHOTONS}

The most important message emerging from the IceCube measurements of the high-energy cosmic neutrino flux is not apparent yet: the prominent role of hadrons relative to leptons in the high-energy universe. As highlighted in the introduction, photons are inevitably produced in association with neutrinos when accelerated cosmic rays produce neutral and charged pions in interactions with target material in the vicinity of the accelerator. Targets include strong radiation fields that may be associated with the accelerator as well as concentrations of matter, such as hydrogen or molecular clouds in their vicinity. The subsequent decay of charged pions via $\pi^{+} \rightarrow \mu^{+}+v_{\mu}$ followed by the decay of the muon $\mu^{+} \rightarrow e^{+}+v_{e}+\bar{v}_{\mu}$ and the chargeconjugate processes produces a flux of high-energy neutrinos. High-energy gamma rays result from the decay of neutral pions, $\pi^{0} \rightarrow \gamma+\gamma$. On average, pionic gamma rays and neutrinos carry $1 / 2$ and $1 / 4$ of the energy of the parent pion, respectively. Using these approximations, one can relate the production rate $Q$ (units of $\mathrm{GeV}^{-1} \mathrm{~s}^{-1}$ ) of neutrinos and gamma rays to their parent pions. For neutrinos, summing over all flavors, the production rate can be related to the one for charged pions as

$$
\sum_{\alpha} E_{v} Q_{v_{\alpha}}\left(E_{v}\right) \simeq 3\left[E_{\pi} Q_{\pi}\left(E_{\pi}\right)\right]_{E_{\pi} \simeq 4 E_{v}} .
$$

Similarly, the production rate of pionic gamma rays is related to the one for neutral pions as

$$
E_{\gamma} Q_{\gamma}\left(E_{\gamma}\right) \simeq 2\left[E_{\pi} Q_{\pi^{0}}\left(E_{\pi}\right)\right]_{E_{\pi} \simeq 2 E_{\gamma}} .
$$

The relative production rates of pionic gamma rays and neutrinos only depend on the ratio of charged-to-neutral pions produced in cosmic-ray interactions, denoted by $K_{\pi}$. In photohadronic interactions, the production is dominated by the production of the $\Delta$ resonance: $p+\gamma \rightarrow \Delta^{+} \rightarrow \pi^{0}+p$ and $p+\gamma \rightarrow \Delta^{+} \rightarrow$ $\pi^{+}+n$. These channels produce charged and neutral pions with probabilities $2 / 3$ and $1 / 3$, respectively. However, the additional contribution of non-resonant pion production modifies the ratios to approximately $1 / 2$ and $1 / 2$. In contrast, if neutrinos originate in the interactions of a hadronic beam with matter, e.g., hydrogen or molecular clouds, equal numbers of pions of all three charges originate: $p+p \rightarrow N_{\pi}\left[\pi^{0}+\pi^{+}+\pi^{-}\right]+X$, where $N_{\pi}$ is the pion multiplicity. Therefore, $K_{\pi} \simeq 2$ for hadronuclear interaction, while $K_{\pi} \simeq 1$ for photohadronic process (Ahlers and Halzen, 2018). We can combine Equations (1) and (2) to derive a direct relation between the pionic gamma-ray and neutrino production rates:

$$
\frac{1}{3} \sum_{\alpha} E_{\nu}^{2} Q_{v_{\alpha}}\left(E_{\nu}\right) \simeq \frac{K_{\pi}}{4}\left[E_{\gamma}^{2} Q_{\gamma}\left(E_{\gamma}\right)\right]_{E_{\gamma}=2 E_{\nu}} .
$$

The pre-factor $1 / 4$ accounts for the energy ratio $\left\langle E_{\nu}\right\rangle /\left\langle E_{\gamma}\right\rangle \simeq 1 / 2$ and the two gamma rays produced in the neutral pion decay. 


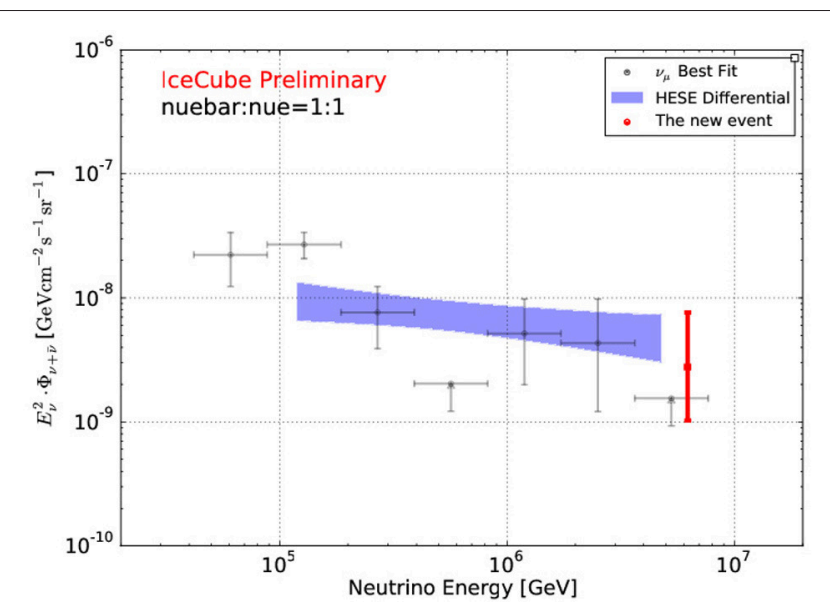

FIGURE 2 | The IceCube diffuse cosmic neutrino spectrum. The best fit to the spectrum of neutrinos observed through the Earth is compared to the unfolded spectrum of events starting inside the detector. Also shown is the flux corresponding to a single candidate event (red) attributed to the Glashow resonance. Figure from Lu (2018).

Note that this relation relates pionic neutrinos and gamma rays without any reference to the cosmic ray beam that initiates the interactions; it simply reflects the fact that a neutral pion produces two gamma rays for every charged pion producing a $v_{\mu}+\bar{v}_{\mu}$ pair, which cannot be separated by current experiments.

Before applying this relation to data, one must take into account the fact that, unlike neutrinos, gamma rays would get absorbed and cascade to lower energies, as they interact with photons of the cosmic microwave background before reaching Earth. If the source is not transparent, intrinsic absorption would also result in lower energy gamma rays leaving the source. The resulting electromagnetic shower subdivides the initial photon energy, leading to multiple photons in the range of $\mathrm{GeV}$ to $\mathrm{TeV}$ energies by the time the gamma rays arrive at Earth (Protheroe and Stanev, 1993; Ahlers et al., 2010).

As described, pionic gamma rays should accompany neutrinos at the site of production. However, since they undergo EBL absorption, they will appear at lower energies. As an illustration, the gamma ray flux accompanying the IceCube diffuse cosmic neutrino flux is described by a simple power law with spectral index of -2.19 , which describes the data obtained from both analyses, above energy of $100 \mathrm{TeV}$. The result is shown for $K_{\pi} \simeq$ 2 by the blue lines in Figure 3. The high-energy cosmic neutrino flux (blue dashed line) follows this initial CR spectrum. Note, however, the observable flux of gamma rays at Earth (blue solid lines) gets strongly attenuated at energies above $100 \mathrm{GeV}$ by interactions with EBL (Ahlers and Halzen, 2018).

The overall normalization of the emission has been chosen not to exceed the isotropic gamma ray background observed by the Fermi satellite (blue data). Interestingly, the high-energy cosmic neutrino flux above $100 \mathrm{TeV}$ shown in Figure 3 saturates this limit. Moreover, the starting event data that extends to lower energies, below $100 \mathrm{TeV}$, are only marginally consistent with the upper bound (blue dashed line). This high intensity of the neutrino flux at lower energies compared to the isotropic gamma-ray background might indicate that these sources are more efficient neutrino than gamma-ray sources (Murase et al., 2016; Bechtol et al., 2017). The similar energy densities of the extragalactic gamma-ray flux observed by Fermi and the highenergy cosmic neutrino flux suggest that, rather than detecting some exotic sources, it is more likely that IceCube to a large extent observes the same universe that conventional astronomy does. Clearly, an extreme universe modeled exclusively on the basis of electromagnetic processes is no longer realistic. We must conclude that a large fraction, possibly most, of the energy in the non-thermal universe originates in hadronic processes, indicating a larger role than previously thought.

Interestingly, the common energy density of photons and neutrinos is also comparable to that of the ultra-high-energy extragalactic cosmic rays above $10^{9} \mathrm{GeV}$ ) (Aab et al., 2015) (green data). A scenario where the high-energy neutrinos observed at IceCube could actually originate in the same sources could be realized as follows: the cosmic ray sources can be embedded in environments that act as "storage rooms" for cosmic rays with energies far below the "ankle" $\left(E_{\mathrm{CR}} \ll 1 \mathrm{EeV}\right)$. This energydependent confinement is obtained by cosmic ray diffusion in magnetic fields. Gamma rays and neutrinos are produced through collisions with gas. Provided that the conditions are met, this mechanism will be so efficient that the total energy stored in cosmic rays below the ankle is converted to that of gamma rays and neutrinos. These "calorimetric" conditions can be achieved, for instance, in starburst galaxies (Loeb and Waxman, 2006) or galaxy clusters (Berezinsky et al., 1997). The qualitatively equal energy in cosmic rays and neutrinos is suggestive of a common origin within the large measurement errors. Additionally, it is further affected by the assumptions on the injected spectrum, the composition of the extragalactic cosmic rays, and, additionally, the transition energy from the Galactic to extragalactic component; see (Globus et al., 2015; Unger et al., 2015; Aab et al., 2017; Kachelrieß et al., 2017) for details.

The extragalactic gamma ray background observed by Fermi (Ackermann et al., 2015) has contributions from identified sources, mostly blazars, on top of an isotropic gamma ray background (IGRB) shown in Figure 3. Furthermore, this diffuse contribution is expected to result from the emission from the same class of gamma ray sources that are individually below Fermi's point-source detection threshold (see, e.g., Di Mauro and Donato, 2015). This speculation received further support when IceCube developed methods for performing real-time multiwavelength observations (Aartsen et al., 2016b, 2017d) that led to the evidence for identification of a distant flaring blazar as a cosmic ray accelerator in a multimessenger campaign launched by a $290 \mathrm{TeV}$ energy neutrino detected from the constellation of Orion on September 22, 2017 (Aartsen et al., 2018a).

\section{THE FIRST TRULY MULTIMESSENGER CAMPAIGN}

Neutrinos only originate in environments where protons are accelerated to produce pions and other particles that decay into neutrinos. Neutrinos can thus exclusively pinpoint cosmic 


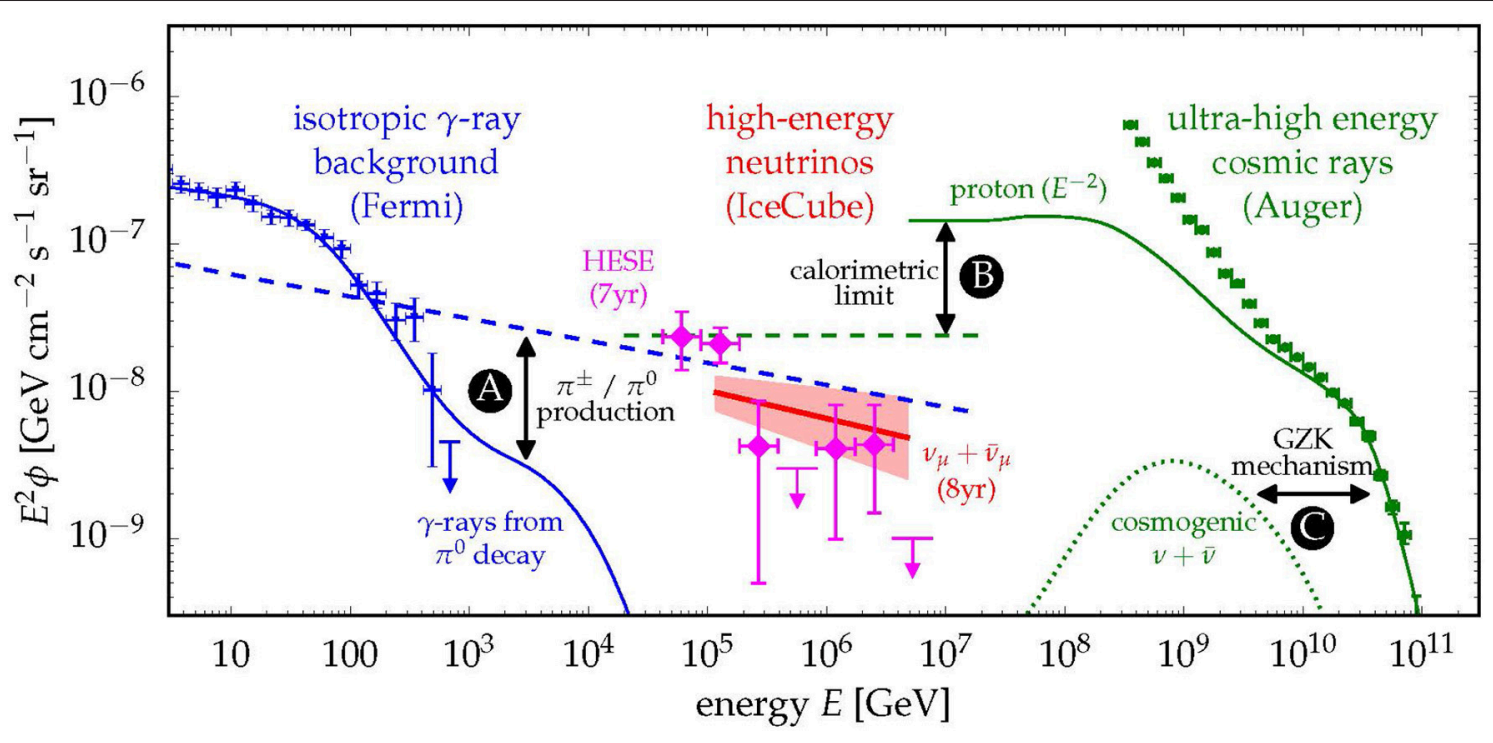

FIGURE 3 | The spectral flux ( $\phi$ ) of neutrinos inferred from the 8-year upgoing track analysis (red solid line) with $1 \sigma$ uncertainty range (shaded range) and the 7-year HESE analysis (magenta data) compared to the flux of unresolved extragalactic gamma ray sources (Ackermann et al., 2015) (blue data) and ultra-high-energy cosmic rays (Aab et al., 2015) (green data). We highlight the various multimessenger interfaces: (A) The joined production of charged pions ( $\pi^{ \pm}$) and neutral pions $\left(\pi^{0}\right)$ in cosmic-ray interactions leads to the emission of neutrinos (dashed blue) and gamma rays (solid blue), respectively. (B) Cosmic ray emission models (solid green) of the most energetic cosmic rays imply a maximal flux (calorimetric limit) of neutrinos from the same sources (green dashed). (C) The same cosmic ray model predicts the emission of cosmogenic neutrinos from the collision with cosmic background photons (GZK mechanism). Figure from Ahlers and Halzen (2018), permission: Elsevier.

ray accelerators, and this is exactly what one neutrino did on September 22, 2017.

IceCube detects muon neutrinos, a type of neutrino that leaves a well-reconstructed track in the detector roughly every $5 \mathrm{~min}$. Most of them are low-energy neutrinos produced in the Earth's atmosphere, which are of interest for studying the neutrinos themselves, but are a persistent background when doing neutrino astronomy. In 2016, IceCube installed an online filter that selects from this sample, in real time, very high energy neutrinos that are likely to be of cosmic origin (Aartsen et al., 2017d). We reconstruct their energy and celestial coordinates, typically in less than $1 \mathrm{~min}$, and distribute the information automatically via the Gamma-ray Coordinate Network to a group of telescopes around the globe and in space for follow-up observations. These telescopes look for electromagnetic radiation from the arrival direction of the neutrino, searching for coincident emission that can reveal its origin.

The tenth such alert (Kopper and Blaufuss, 2017), IceCube170922A, on September 22, 2017, reported a well-reconstructed muon neutrino with an energy of $290 \mathrm{TeV}$ and, therefore, with a high probability of originating in an astronomical source. The Fermi telescope detected a flaring blazar aligned with the cosmic neutrino within 0.06 degrees. The source is a known blazar, a supermassive black hole spitting out high-energy particles in twin jets aligned with its rotation axis which is directed at Earth. This blazar, TXS $0506+056$, had been relatively poorly studied until now, although it was identified by EGRET as the preeminent source for observation of two photons with energies above $40 \mathrm{GeV}$ (Dingus and Bertsch, 2001). Observations triggered by the neutrino alert yielded a treasure trove of multiwavelength data. An optical telescope eventually measured its distance (Paiano et al., 2018), which was found at redshift of 0.34. Its large distance points to a special galaxy, which sets it apart from the ten-times-closer blazars, such as the Markarian sources that dominate the extreme gamma-ray sky observed by NASA's Fermi satellite. Getting all the elements of this multimessenger observation together has been challenging. Attempts to explain the broadband multiwavelength spectrum of the source indicate a subdominant hadronic emission for the coincident observations. The hadronic contribution to the highenergy emission is constrained by the X-ray flux expected in conventional models for blazars. For details, see (Gokus et al., 2018; Keivani et al., 2018; Murase et al., 2018; Sahakyan, 2018; Zhang et al., 2018; Cerruti et al., 2019; Gao et al., 2019).

TXS $0506+056$ was originally flagged by the Fermi (Tanaka et al., 2017) and Swift (Keivani et al., 2017) satellite telescopes. Follow-up observations with the MAGIC air Cherenkov telescope (Mirzoyan, 2017) identified it as a rare very high energy blazar with the potential to produce the very high energy neutrino detected by IceCube. The source was subsequently scrutinized in X-ray, optical, and radio wavelengths. This is a first, truly multimessenger observation: none of the instruments could have made this breakthrough independently. In total, more than 20 telescopes observed the flaring blazar as a highly variable source in a high state (Aartsen et al., 2018a).

It is important to realize that nearby blazars like the Markarian sources are at a redshift that is ten times smaller, and therefore TXS 0506+056, with a similar flux despite the greater distance, is one of the most luminous sources in the Universe. It likely belongs to a special class of blazars that accelerate proton beams as revealed by the neutrino. This explains the fact that a variety of previous attempts to associate the arrival directions of cosmic 
neutrinos with the bulk of Fermi blazars (Aartsen et al., 2017a) have failed.

Informed by the multimessenger campaign, IceCube searched its archival neutrino data up to and including October 2017 in the direction of IC-170922A using the likelihood routinely used in previous searches. This revealed a spectacular burst of over a dozen high-energy neutrinos in 110 days in the 2014-2015 data with a spectral index similar to the one observed for the diffuse cosmic neutrino spectrum (Aartsen et al., 2018b).

It is worth mentioning that the AGILE collaboration, which operates an orbiting X-ray and gamma ray telescope, reported a day-long flare in the direction of a previous neutrino alert sent on July 31, 2016 (Lucarelli et al., 2017). The flare occurred more than one day before the time of the alert. In light of the rapid daily variations observed near the peak emission of the TXS $0506+056$ flare at the time of IC-170922A, this may well be a genuine coincidence.

\section{FLARING SOURCES AND THE HIGH-ENERGY NEUTRINO FLUX}

As we will show further on, it is clear that a source that produces more than a dozen high-energy neutrinos in only 110 days cannot be a routine blazar in the Fermi sky. This single flare in 20142015 dominates the flux of the source over the 9.5 years of archival IceCube data, leaving IC-170922A as a less luminous second flare in the sample. With the density of photons in the target required to produce the neutrinos, the source cannot be transparent to the high-energy photons routinely observed by Fermi. This, as well as the detection of this extraordinary large neutrino flux from a source at a redshift of 0.34 , when photonbright blazars are observed at distances closer by one order of magnitude, suggests that TXS $0506+056$ belongs to a special class of sources, those that produce cosmic rays. We will show next that a subset of about $5 \%$ of all blazars can accommodate the diffuse cosmic neutrino flux observed by IceCube assuming they burst once in 10 years at the level of TXS 0506+056 in 2014 . We have already pointed out in the previous section that the energy density of this flux is similar to that in the highest energy cosmic rays.

The all-sky flux of high-energy neutrinos from a fraction $(\mathcal{F})$ of a class of sources with density $\rho$, neutrino luminosity $L_{v}$, and episodic emission of flares of duration $\Delta t$ over a total observation time $T$ is

$$
\sum_{\alpha} E_{v}^{2} \frac{d N_{v}}{d E_{v}}=\frac{1}{4 \pi} \frac{c}{H_{0}} \xi_{z} L_{v} \rho \mathcal{F} \frac{\Delta t}{T} .
$$

where $\xi_{z}$ is a factor of order unity that parameterizes the integration over the redshift evolution of the sources (Halzen et al., 2018). Applying this relation to the 2014 TXS 0506+056 burst that dominates the flux over the 9.5 years of neutrino observations, yields

$$
\begin{aligned}
3 \times 10^{-11} \mathrm{TeVcm}^{-2} \mathrm{~s}^{-1} \mathrm{sr}^{-1}= & \frac{\mathcal{F}}{4 \pi} \frac{c}{H_{0}}\left(\frac{\xi_{z}}{0.7}\right)\left(\frac{L_{v}}{1.2 \times 10^{47} \mathrm{erg} / \mathrm{s}}\right) \\
& \left(\frac{\rho}{1.5 \times 10^{-8} \mathrm{Mpc}^{-3}}\right)\left(\frac{\Delta t}{110 \mathrm{~d}} \frac{10 \mathrm{yr}}{T}\right)
\end{aligned}
$$

a relation which is satisfied for $\mathcal{F} \sim 0.05$. In summary, a special class of BL Lac blazars that undergo $\sim 110$-day duration flares like TXS $0506+056$ once every 10 years accommodates the observed diffuse flux of high-energy cosmic neutrinos. The class of such neutrino-flaring sources represents $5 \%$ of the sources. The argument implies the observation of roughly 100 muon neutrinos per year. This is exactly the flux of cosmic neutrinos that corresponds to the $E^{-2.19}$ diffuse flux measured above $100 \mathrm{TeV}$. (Note that the majority of these neutrinos cannot be separated from the atmospheric background, leaving us with the reduced number of very high energy events discussed in the previous sections). As previously discussed, the energetics of the cosmic neutrinos is matched by the energy of the highest energy cosmic rays, with

$$
\frac{1}{3} \sum_{\alpha} E_{v}^{2} \frac{d N_{v}}{d E_{v}} \simeq \frac{c}{4 \pi}\left(\frac{1}{2}\left(1-e^{-f_{\pi}}\right) \xi_{z} t_{H} \frac{d E}{d t}\right)
$$

The cosmic rays' injection rate $d E / d t$ above $10^{16} \mathrm{eV}$ is $(1-$ 2) $\times 10^{44} \mathrm{erg}^{\mathrm{Mpc}}{ }^{-3} \mathrm{yr}^{-1}$ (Ahlers and Halzen, 2012; Katz et al., 2013). From Equation (5) it follows that the energy densities match for a pion production efficiency of the neutrino source of $f_{\pi} \gtrsim 0.4$. This high efficiency requirement is consistent with the premise that a special class of efficient sources is responsible for producing the high-energy cosmic neutrino flux seen by IceCube. The sources must contain sufficient target density in photons, possibly protons, to generate the large value of $f_{\pi}$. It is clear that the emission of flares producing the large number of cosmic neutrinos detected in the 2014 burst must correspond to major accretion events onto the black hole lasting a few months. The pionic photons will lose energy in the source and the neutrino emission is not accompanied by a flare as was the case for the 2017 event (See Halzen et al., 2018 for details); the Fermi data, consistent with the scenario proposed, reveal photons with energies of tens of $\mathrm{GeV}$, but no enhancement in gamma ray activity (Garrappa et al., 2018).

In the modeling of hadronic blazars, it is typically assumed that the interaction of accelerated protons with photons is responsible for generating pions that decay into neutrinos. The interaction is dominated by the $\Delta$-resonance, with $p \gamma \rightarrow \Delta \rightarrow$ $\pi N$. Accelerated cosmic rays may interact with photons in the jet or with stationary photons provided by the accretion disk. Assuming the former case, the efficiency for producing pions depends on the target photons energy, the Lorentz factor of the jet, the luminosity of the photons, and the duration of the flare:

$$
f_{\pi} \simeq \frac{L_{\gamma}}{E_{\gamma}} \frac{1}{\Gamma^{2} \Delta t} \frac{3 \sigma_{\Delta}\left\langle x_{p \rightarrow \pi}\right\rangle}{4 \pi c^{2}}
$$

Since TXS $0506+056$ is an intermediate synchrotron-peaked blazar, we consider the target photon at the synchrotron energy peak, $10 \mathrm{eV}$, to constrain the parameter space for the 
pion efficiency:

$$
\begin{array}{r}
f_{\pi} \gtrsim 0.4 \simeq\left(\frac{L_{\gamma}}{2 \times 10^{46} \mathrm{erg} / \mathrm{s}}\right)\left(\frac{10 \mathrm{eV}}{E_{\gamma}}\right)\left(\frac{1}{\Gamma^{2}}\right) \\
\left(\frac{110 \mathrm{~d}}{\Delta t}\right)\left(\frac{3 \sigma_{\Delta}\left\langle x_{p \rightarrow \pi}\right\rangle}{4 \pi c^{2}}\right) .
\end{array}
$$

This suggests that sources with a small Lorentz factor and UV luminosity of $\mathcal{O}\left(10^{46}\right)$ can satisfy the requirements for producing the high-energy cosmic neutrinos observed. This level of UV luminosity is compatible with the Swift-UVOT measurement reported by Padovani et al. (2018). Similar values for UV luminosity were also reported in Krauß et al. (2014). In addition, the study of radio structure of TXS 0506+056 suggests the feasibility of a small Lorentz factor (Kun et al., 2018). The case for a small Lorentz factor also follows arguments made in the context of a tentative observation of high-energy neutrinos by AMANDA in coincidence with a flare of 1ES 1959+560 in 2002 (Halzen and Hooper, 2005).

A key question is whether the neutrino and gamma-ray spectra for the 2014 neutrino burst from TXS 0506+056 satisfy the multimessenger relationship introduced in section 2 . With the low statistics of the very high energy gamma-ray measurements during the burst period, the energetics is a more robust measure for evaluating the connection, especially because the source is opaque to high-energy gamma rays, as indicated by the large value of $f_{\pi}$, and the pionic gamma rays will lose energy inside the source before cascading in the microwave photon background; for details, see (Halzen et al., 2018).

The gamma-ray opacity can be related to $f_{\pi}$ as

$$
\tau_{\gamma \gamma} \approx \frac{\eta_{\gamma \gamma} \sigma_{\gamma \gamma}}{\eta_{p \gamma} \hat{\sigma}_{p \gamma}} f_{\pi}
$$

where $\hat{\sigma}_{p \gamma} \sim 0.7 \times 10^{-28} \mathrm{~cm}^{2}$ and $\sigma_{\gamma \gamma} \simeq 6.65 \times 10^{-25} \mathrm{~cm}^{2}$ (Murase et al., 2016). We assume $\eta_{\gamma \gamma} \sim 0.1$ and $\eta_{p \gamma} \simeq$ 1. This implies $\tau_{\gamma \gamma} \simeq \mathcal{O}(100)$ for $f_{\pi} \gtrsim 0.4$. This high opacity will make it impossible for the very high energy pionic gamma rays, with energies similar to those of the neutrinos, to leave the source.

Absorption and interactions intrinsic to the source due to the high opacity, followed by the interaction with EBL, will result in a suppressed gamma-ray flux, and hence a gamma-ray flare is not expected as the source is a highly efficient neutrino emitter (Halzen et al., 2018). This is consistent with the Fermi observation of the source during the neutrino flare in 2014 (Garrappa et al., 2018; Padovani et al., 2018). Potential hardening reported by Padovani et al. (2018) could be explained in this picture (see Halzen et al., 2018). The absence of gamma rays during a neutrino burst could also be a result of secondary gamma rays spreading in a larger opening angle compared to the original beam; see (Neronov and Semikoz, 2002) for details.

It is worth noting that this model for the diffuse neutrino flux clarifies why earlier attempts to associate it with the bulk of blazars were unsuccessful and why the limits built on source population and non-detection of a steady source do not apply.
Clearly, the time-integrated studies are not applicable to timedependent sources. Moreover, with a subclass of more energetic sources with lower density responsible for the diffuse flux, the constraints on blazars obtained from the relation between the point source limits and the diffuse flux are mitigated. The idea of a subclass of sources with strong emission is further supported by the rapid evolution of sources; see (Neronov and Semikoz, 2018) for details. These speculations are qualitative and may be premature, but the hope is that multimessenger astronomy will provide us with more clues after the breakthrough event of September 22, 2017, which generated an unmatched data sample over all wavelengths of the electromagnetic spectrum of a likely cosmic ray accelerator.

\section{SUMMARY}

Our main conclusion is that this aspect of multimessenger astronomy is likely to be subtle. The spectacular neutrino burst in 2014-2015 must originate from a source that is obscured to high-energy photons and leaves a signature in the Fermi data that is not straightforward and likely to change on an event by event basis. On the other hand, the intense photon flare in 2017 was accompanied by a single very high energy neutrino, most likely a fluctuation from a much lower flux. Getting all the elements of this puzzle to fit together will not be easy, especially because we are missing the rich spectrum of multiwavelength observations in 2014 that were the result of the extensive multimessenger campaign at all wavelengths in September 2017. We need more neutrinos from multiple and more sensitive detectors, and we need a future gamma-ray mission with uninterrupted all-sky coverage.

Unlike the previous SN1987A and GW170817 multimessenger events, this event could not have been observed with a single instrument. Without the initial coincident observation, IC-170922A would be just one more of the few hundred cosmic neutrinos detected by IceCube and the accompanying radiation just one more flaring blazar observed by Fermi-LAT. Neutrino astronomy was born with a supernova in 1987. Thirty years later, this recent event involves neutrinos that are tens of millions of times more energetic and are from a source a hundred thousand times more distant.

\section{AUTHOR CONTRIBUTIONS}

The introductory material was mostly written by $\mathrm{FH}$, the more substantive material about the multimessenger event and the interpretation of the data was a collaborative effort of $\mathrm{AK}$ and $\mathrm{FH}$.

\section{ACKNOWLEDGMENTS}

The authors are supported in part by NSF under grants PLR1600823 and PHY-1607644 and by the University of Wisconsin Research Council with funds granted by the Wisconsin Alumni Research Foundation. 


\section{REFERENCES}

Aab, A., Abreu, P., Aglietta, M., Ahn, E. J., Al Samarai, I., Albuquerque, I. F. M., et al. (2015). "The Pierre Auger Observatory: Contributions to the 34th International Cosmic Ray Conference (ICRC 2015)," in Proceedings, 34th International Cosmic Ray Conference (ICRC 2015) (The Hague).

Aab, A., Abreu, P., Aglietta, M., Al Samarai, I., Albuquerque, I. F. M., Allekotte, I. et al. (2017). Inferences on mass composition and tests of hadronic interactions from 0.3 to $100 \mathrm{EeV}$ using the water-Cherenkov detectors of the Pierre Auger Observatory. Phys. Rev. D96:122003. doi: 10.1103/PhysRevD.96.122003

Aartsen, M. G., Abbasi, R., Abdou, Y., Ackermann, M., Adams, J., Aguila, J. A., et al. (2013a). First observation of PeV-energy neutrinos with IceCube. Phys. Rev. Lett. 111:021103. doi: 10.1103/PhysRevLett.111.021103

Aartsen, M. G., Abbasi, R., Abdou, Y., Ackermann, M., Adams, J., Aguila, J. A., et al. (2013b). Measurement of South Pole ice transparency with the IceCube LED calibration system. Nucl. Instrum. Methods A711, 73-89. doi: 10.1016/j.nima.2013.01.054

Aartsen, M. G., Abbasi, R., Abdou, Y., Ackermann, M., Adams, J., Aguilar, J. A., et al. (2013c). Evidence for high-energy extraterrestrial neutrinos at the IceCube detector. Science 342:1242856. doi: 10.1126/science. 1242856

Aartsen, M. G., Abbasi, R., Ackermann, M., Adams, J., Aguilar, J. A., Ahlers, M., et al. (2014). Energy reconstruction methods in the IceCube neutrino telescope. JINST 9:P03009. doi: 10.1088/1748-0221/9/03/P03009

Aartsen, M. G., Abraham, K., Ackermann, M., Adams, J., Aguilar, J. A., Ahlers, M., et al. (2015). Evidence for astrophysical muon neutrinos from the Northern sky with IceCube. Phys. Rev. Lett. 115:081102. doi: 10.1103/PhysRevLett.115.081102

Aartsen, M. G., Abraham, K., Ackermann, M., Adams, J., Aguilar, J. A., Ahlers, M., et al. (2016a). Observation and characterization of a Cosmic Muon Neutrino Flux from the Northern Hemisphere using six years of IceCube data. Astrophys. J. 833:3. doi: 10.3847/0004-637X/833/1/3

Aartsen, M. G., Abraham, K., Ackermann, M., Adams, J., Aguilar, J. A., Ahlers, M., et al. (2016b). Very high-energy gamma-ray follow-up program using Neutrino triggers from IceCube. JINST 11:P11009. doi: 10.1088/1748-0221/11/11/P11009

Aartsen, M. G., Abraham, K., Ackermann, M., Adams, J., Aguilar, J. A., Ahlers, M., et al. (2017a). The contribution of Fermi-2LAC blazars to the diffuse TeV-PeV neutrino flux. Astrophys. J. 835:45. doi: 10.3847/1538-4357/835/1/45

Aartsen, M. G., Ackerman, M., Adams, J., Aguilar, J. A., Ahlers, M., Ahrens, M., et al. (2018a). Multimessenger observations of a flaring blazar coincident with high-energy neutrino IceCube-170922A. Science 361:eaat1378. doi: $10.1126 /$ science.aat1378

Aartsen, M. G., Ackermann, M., Adams, J., Aguilar, J. A., Ahlers, M., Ahrens, M., et al. (2017b). The IceCube neutrino observatory - Contributions to ICRC 2017 part II: properties of the atmospheric and astrophysical neutrino flux. Available online at: https://arxiv.org/abs/1710.01191

Aartsen, M. G., Ackermann, M., Adams, J., Aguilar, J. A., Ahlers, M., Ahrens, M., et al. (2017c). The IceCube neutrino observatory: instrumentation and online systems. JINST 12:P03012. doi: 10.1088/1748-0221/12/03/P03012

Aartsen, M. G., Ackermann, M., Adams, J., Aguilar, J. A., Ahlers, M., Ahrens, M., et al. (2017d). The IceCube realtime alert system. Astropart. Phys. 92, 30-41. doi: 10.1016/j.astropartphys.2017.05.002

Aartsen, M. G., Ackermann, M., Adams, J., Aguilar, J. A., Ahlers, M., Ahrens, M., et al. (2018b). Neutrino emission from the direction of the blazar TXS $0506+056$ prior to the IceCube-170922A alert. Science 361, 147-151. doi: $10.1126 /$ science.aat 2890

Ackermann, M., Ajello, M., Albert, A., Atwood, W. B., Baldini, L., Ballet, J., et al. (2015). The spectrum of isotropic diffuse gamma-ray emission between 100 $\mathrm{MeV}$ and $820 \mathrm{GeV}$. Astrophys. J. 799:86. doi: 10.1088/0004-637X/799/1/86

Ahlers, M., Anchordoqui, L., Gonzalez-Garcia, M., Halzen, F., and Sarkar, S. (2010). GZK Neutrinos after the Fermi-LAT diffuse photon flux measurement. Astropart. Phys. 34, 106-115. doi: 10.1016/j.astropartphys.2010.06.003

Ahlers, M., and Halzen, F. (2012). Minimal cosmogenic neutrinos. Phys. Rev. D86:083010. doi: 10.1103/PhysRevD.86.083010

Ahlers, M., and Halzen, F. (2018). Opening a new window onto the Universe with IceCube. Prog. Part. Nucl. Phys. 102, 73-88. doi: 10.1016/j.ppnp.2018.05.001

Bechtol, K., Ahlers, M., Di Mauro, M., Ajello, M., and Vandenbroucke, J. (2017). Evidence against star-forming galaxies as the dominant source of IceCube neutrinos. Astrophys. J. 836:47. doi: 10.3847/1538-4357/836/1/47
Berezinsky, V., Blasi, P., and Ptuskin, V. (1997). Clusters of galaxies as a storage room for cosmic rays. Astrophys J. 487, 529-535. doi: 10.1086/304622

Cerruti, M., Zech, A., Boisson, C., Emery, G., Inoue, S., and Lenain, J. P. (2019). Lepto-hadronic single-zone models for the electromagnetic and neutrino emission of TXS 0506+056. Mon. Notices R. Astron. Soc. 483:L12. doi: $10.1093 / \mathrm{mnrasl} / \mathrm{sly} 210$

Di Mauro, M., and Donato, F. (2015). Composition of the Fermi-LAT isotropic gamma-ray background intensity: emission from extragalactic point sources and dark matter annihilations. Phys. Rev. D91:123001. doi: 10.1103/PhysRevD.91.123001

Dingus, B. L., and Bertsch, D. L. (2001). The highest energy emission detected by egret from blazars. AIP Conf. Proc. 587, 251-255. doi: 10.1063/1. 1419408

Fang, K., and Murase, K. (2018). Linking high-energy cosmic particles by black hole jets embedded in large-scale structures. Phys. Lett., 14:396. doi: 10.1038/s41567-017-0025-4

Gao, S., Fedynitch, A., Winter, W., and Pohl, M. (2019). Modelling the coincident observation of a high-energy neutrino and a bright blazar flare. Nat. Astron. 3, 88-92. doi: 10.1038/s41550-018-0610-1

Garrappa, S. (2018). Gamma ray counterparts to IceCube high-energy tracks. in 8th International Fermi Symposium. Baltimore, MD.

Globus, N., Allard, D., and Parizot, E. (2015). A complete model of the cosmic ray spectrum and composition across the Galactic to extragalactic transition. Phys. Rev. D92:021302. doi: 10.1103/PhysRevD.92.021302

Gokus, A., Richter, S., Spanier, F., Kreter, M., Kadler, M., Mannheim, K., and Wilms, J. (2018). Decomposing blazar spectra into lepto-hadronic emission components. Availble online at: https://arxiv.org/abs/1808.05540

Halzen, F., and Hooper, D. (2005). High energy neutrinos from the TeV blazar 1ES 1959+650. Astropart. Phys. 23, 537-542. doi: 10.1016/j.astropartphys.2005.03.007

Halzen, F., Kheirandish, A., Weisgarber, T., and Wakely, S. P. (2018). On the neutrino flares from the direction of TXS 0506+056. Astrophys. J. 874, L9. doi: 10.3847/2041-8213/ab0d27

Kachelrieß, M., Kalashev, O., Ostapchenko, S., and Semikoz, D. V. (2017). Minimal model for extragalactic cosmic rays and neutrinos. Phys. Rev. D96:083006. doi: 10.1103/PhysRevD.96.083006

Katz, B., Waxman, E., Thompson, T., and Loeb, A. (2013). The energy production rate density of cosmic rays in the local universe is $\sim 10^{44-45} \mathrm{erg} \mathrm{Mpc}^{-3} \mathrm{yr}^{-1}$ at all particle energies. Available online at: https://arxiv.org/abs/1311.0287

Katz, U. F., and Spiering, C. (2012). High-Energy Neutrino Astrophysics: Status and Perspectives. Prog. Part. Nucl. Phys. 67, 651-704. doi: 10.1016/j.ppnp.2011.12.001

Keivani, A., Murase, K., Petropoulou, M., Fox, D. B., Cenko, S. B., Chaty, S., et al. (2018). A multimessenger picture of the flaring blazar TXS 0506+056: implications for high-energy neutrino emission and cosmic ray acceleration. Astrophys. J. 864:84. doi: 10.3847/1538-4357/aad59a

Keivani, P. A., Evans, A., Kennea, J. A., Fox, D. B., Cowen, D. F., Osborne, J. P., et al. (2017). "Further Swift-XRT observations of IceCube 170922A". in The Astronomer's Telegram, 10792.

Kopper, C., and Blaufuss, E. (2017). "IceCube-170922A - IceCube observation of a high-energy neutrino candidate event.," in GRB Coordinates Network, Circular Service, No. 21916, \#1 (2017), 21916.

Krauß, F., Kadler, M., Mannheim, K., Schulz, R., Trüstedt, J., Wilms, J., et al. (2014). TANAMI blazars in the IceCube PeV Neutrino fields. Astron. Astrophys. 566:L7. doi: 10.1051/0004-6361/201424219

Kun, E., Biermann, P. L., and Gergely, L. (2018). VLBI radio structure and corebrightening of the high-energy neutrino emitter TXS 0506+056. Mon. Not. Roy. Astron. Soc. 483, L42-L46. doi: 10.1093/mnrasl/sly216

Loeb, A., and Waxman, E. (2006). The cumulative background of high energy neutrinos from starburst galaxies. JCAP 0605:003. doi: $10.1088 / 1475-7516 / 2006 / 05 / 003$

Lu, L. (2018). "Recent IceCube results - evidences of neutrino emission from the blazar TXS 0506+056 and searches for Glashow resonance." Contribution to Ultra High Energy Cosmic Rays 2018. Paris, France.

Lucarelli, F., Pittori, C., Verrecchia, F., Donnarumma, I., Tavani, M., Bulgarelli, A., et al. (2017). AGILE detection of a candidate gamma-ray precursor to the ICECUBE-160731 neutrino event. Astrophys. J. 846:121. doi: $10.3847 / 1538-4357 /$ aa81c8 
Mirzoyan, R. (2017). "First-time detection of VHE gamma rays by MAGIC from a direction consistent with the recent EHE neutrino event IceCube-170922A," in The Astronomer's Telegram, 10817.

Murase, K., Guetta, D., and Ahlers, M. (2016). Hidden cosmic-ray accelerators as an origin of TeV-PeV cosmic neutrinos. Phys. Rev. Lett. 116:071101. doi: 10.1103/PhysRevLett.116.071101

Murase, K., Oikonomou, F., and Petropoulou, M. (2018). Blazar flares as an origin of high-energy cosmic neutrinos? Astrophys. J. 865:124. doi: $10.3847 / 1538-4357 /$ aada00

Neronov, A., and Semikoz, D. V. (2018). Self-consistent model of extragalactic neutrino flux from evolving blazar population. Available online at: https://arxiv. org/abs/1811.06356

Neronov, A. Y., and Semikoz, D. V. (2002). Which blazars are neutrino loud? Phys. Rev. D66:123003. doi: 10.1103/PhysRevD.66.123003

Padovani, P., Giommi, P., Resconi, E., Glauch, T., Arsioli, B., Sahakyan, N., et al. (2018). Dissecting the region around IceCube-170922A: the blazar TXS 0506+056 as the first cosmic neutrino source. Mon. Notices R. Astron. Soc. 480:192. doi: 10.1093/mnras/sty 1852

Paiano, S., Falomo, R., Treves, A., and Scarpa, R. (2018). The redshift of the BL Lac object TXS 0506+056. Astrophys. J. 854:L32. doi: 10.3847/2041-8213/ aaad5e

Protheroe, R. J. and Stanev, T. (1993). Electron-photon cascading of very highenergy gamma-rays in the infrared background. Mon. Notices R. Astron. Soc. $264,191-200$.

Sahakyan, N. (2018). Lepto-hadronic $\gamma$-ray and neutrino emission from the jet of TXS 0506+056. Astrophys. J. 866:109. doi: 10.3847/1538-4357/aadade
Taboada, I. (2018). "A View of the Universe with the IceCube and ANTARES Neutrino Telescopes.” Neutrino 2018. Heidelberg, Germany. doi: 10.5281/zenodo.1286919

Tanaka, Y. T., Buson, S., and Kocevski, D. (2017). "Fermi-LAT detection of increased gamma-ray activity of TXS $0506+056$, located inside the IceCube170922A error region," in The Astronomer's Telegram, 10791.

Unger, M., Farrar, G. R., and Anchordoqui, L. A. (2015). Origin of the ankle in the ultrahigh energy cosmic ray spectrum, and of the extragalactic protons below it. Phys. Rev. D92:123001. doi: 10.1103/PhysRevD.92.123001

Yuan, T. (2017). "Improving the angular resolution in icecube cascade reconstruction," in TeV Particle Astrophysics 2017 (TeVPA2017) (Columbus, $\mathrm{OH})$.

Zhang, H., Fang, K., and Li, H. (2018). Variability and optical polarization can probe the neutrino and electromagnetic emission mechanisms of TXS 0506+056. Available online at: https://arxiv.org/abs/1807.11069

Conflict of Interest Statement: The authors declare that the research was conducted in the absence of any commercial or financial relationships that could be construed as a potential conflict of interest.

Copyright (c) 2019 Halzen and Kheirandish. This is an open-access article distributed under the terms of the Creative Commons Attribution License (CC BY). The use, distribution or reproduction in other forums is permitted, provided the original author(s) and the copyright owner(s) are credited and that the original publication in this journal is cited, in accordance with accepted academic practice. No use, distribution or reproduction is permitted which does not comply with these terms. 